\title{
Alterstice
}

Revue internationale de la recherche interculturelle

International Journal of Intercultural Research

Revista International de la Investigacion Intercultural

\section{La philosophie interculturelle. Penser autrement le monde, de Raúl Fornet-Betancourt}

\section{Olivier Ducharme}

Volume 1, numéro 1, 2011

URI : https://id.erudit.org/iderudit/1077596ar

DOI : https://doi.org/10.7202/1077596ar

Aller au sommaire du numéro

Éditeur(s)

Alterstice

ISSN

1923-919X (numérique)

Découvrir la revue

Citer ce compte rendu

Ducharme, O. (2011). Compte rendu de [La philosophie interculturelle. Penser autrement le monde, de Raúl Fornet-Betancourt]. Alterstice, 1(1), 107-110.

https://doi.org/10.7202/1077596ar d'utilisation que vous pouvez consulter en ligne.

https://apropos.erudit.org/fr/usagers/politique-dutilisation/ 


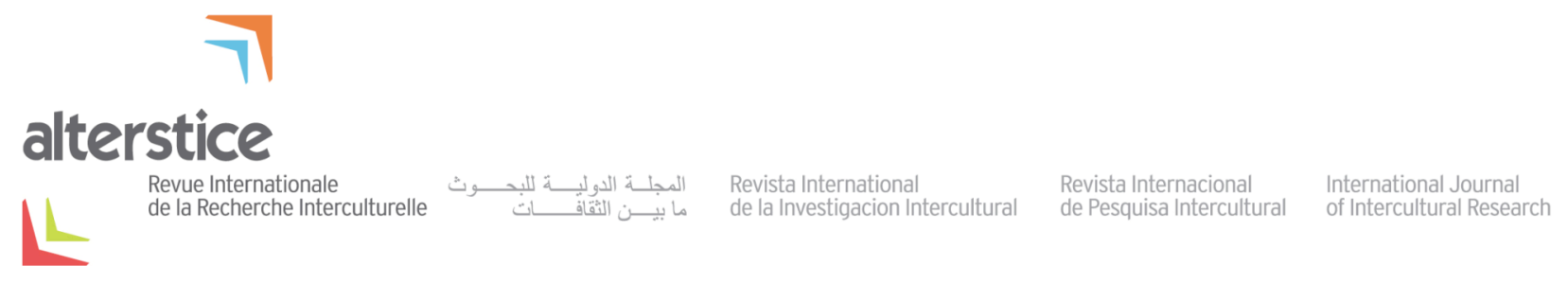

NOTE DE LECTURE

\title{
La philosophie interculturelle. Penser autrement le monde, de Raúl Fornet- Betancourt
}

Olivier Ducharme ${ }^{1}$

\author{
Rattachement de l'auteur \\ ${ }^{1}$ Faculté de philosophie, Université Laval, Québec, Canada
}

\section{Correspondance}

ducharmeolivier@hotmail.com

\section{Références de l'ouvrage :}

Fornet-Betancourt, R. (2011). La philosophie interculturelle. Penser autrement le monde (A. Kasanda, trad.). Paris : Les éditions de l'Atelier. (ISBN : 978-2-7082-4152-7)

\section{Pour citer cet article :}

Ducharme, O. (2011). La philosophie interculturelle. Penser autrement le monde [Note de lecture]. Alterstice, 1(1), 107-110.

La pensée philosophique d'Amérique latine ne reçoit que peu ou même pas du tout d'intérêt de la part de la philosophie continentale de tradition européenne. Les facultés et les départements de philosophie d’Europe et d'Amérique du Nord s'en tiennent à l'étude de leur propre tradition et de leur propre contexte culturel. La lecture de l'ouvrage de Raúl Fornet-Betancourt, La philosophie interculturelle, nous place devant notre propre ignorance de la situation philosophique en Amérique latine. Nous croyons que cette ignorance est partagée par la plupart des philosophes ayant leur racine dans le milieu académique européen et nord-américain. Cet ouvrage nous dévoile le contrepoids ou la partie ombragée d'une Amérique qui cherche à se dire "Amérique " par delà l'Europe, ou du moins, par delà le poids trop grand de la tradition européenne. Fornet-Betancourt cherche à rendre compte de cette Amérique en développant les potentialités d'une « philosophie interculturelle ».

Publié originellement en 2001 en espagnol, et traduit par Albert Kasanda (à qui l'on doit également la traduction de L'éthique de la libération à l'ère de la mondialisation et de l'exclusion d'Enrique Dussel), cet ouvrage est la première traduction complète publiée en français par Fornet-Betancourt. Auteur de plus d'une quinzaine d'ouvrages, FornetBetancourt est directeur du département d'Amérique latine à l'Institut d'Aix-la-Chapelle en Allemagne. Fred Poché écrit à propos de Fornet-Betancourt dans sa préface à La philosophie interculturelle: "Philosophe d'envergure internationale, Raúl Fornet-Betancourt apparaît comme un penseur infatigable entre le Nord et le Sud, un praticien convaincu du dialogue interculturel et un défenseur farouche d'une philosophie engagée face à l'universalisme ethnocentrique occidental et au rouleau compresseur de la globalisation capitaliste. ॥ (p. 5) Malgré ces bons mots, et si l'on tient compte du fait qu'il s'agit de son premier ouvrage traduit en français, nous pouvons conclure que la 
pensée interculturelle développée par Fornet-Betancourt souffre encore aujourd'hui, dans le milieu francophone, d'une méconnaissance. L'affirmation de Fred Poché selon laquelle la publication en français de La philosophie interculturelle " est un événement intellectuel majeur » s'explique facilement du fait qu'il s'agit de la première intrusion importante de la pensée de Fornet-Betancourt dans le milieu francophone.

L'ouvrage de Fornet-Betancourt se veut le parcours programmatique de ce qu'est et de ce que devrait être la philosophie interculturelle. Il faut souligner ici la présence du conditionnel, car il s'agit bien, lorsque FornetBetancourt décrit les lignes directrices de la philosophie interculturelle, d'une visée qui est non sans rappeler les Idées régulatrices kantiennes ou la "démocratie à venir» de Jacques Derrida. Pour Fornet-Betancourt, la philosophie interculturelle se fonde sur une promesse ou sur un avenir dans lequel elle pourra se déployer dans toutes ses possibilités. Comme pour la « démocratie à venir » de Derrida, la philosophie interculturelle est le nom générique d'une promesse qui est à venir. Derrida écrit à propos de la «démocratie à venir » à la toute fin de Politiques de l'amitié :

Car la démocratie reste à venir, c'est là son essence en tant qu'elle reste: non seulement elle restera indéfiniment perfectible, donc toujours insuffisante et future mais, appartenant au temps de la promesse, elle restera toujours, en chacun de ses temps futurs, à venir : même quand il y a la démocratie, celle-ci n'existe jamais, elle n'est jamais présente, elle reste le thème d’un concept non présentable » (Derrida, 1994, p. 339)

Nous croyons lire dans cette description de la "démocratie à venir » derridienne l'un des aspects majeurs de la philosophie interculturelle développée par Fornet-Betancourt. Cette dernière vise la description d'une pratique philosophique "indéfiniment perfectible ", c'est-à-dire une pratique qui est intimement liée à l'avenir et à un processus continuel de développement. La philosophie interculturelle se lie intimement à l'avenir, car sa seule position possible se trouve dans un travail continuel de mise en application de sa visée. L'ethos de libération, qui est au fondement de la philosophie interculturelle, doit demeurer une pratique qui est à jamais ouverte et qui laisse ainsi toute la place au travail futur.

Cette promesse et cette ouverture à l'avenir, qui sont au cœur de la philosophie interculturelle, doivent se lire également dans leur énoncé même. Dans un geste qui rappelle de nouveau Derrida et également la pensée postcoloniale (Homi Bhabha, Gayatri Chakravorty Spivak), la philosophie interculturelle se fonde sur l'entre ou l'inter. Au lieu de situer le noyau dur de la pensée dans le principe d'identité, Fornet-Betancourt le localise dans la différence entre les cultures. Cette relocalisation de la pensée permet ainsi une ouverture de chaque culture à toutes les autres cultures. C'est à ce point de la différence qu'apparaît l'un des concepts-clés de l'ouvrage de Fornet-Betancourt : le dialogue. II s'agit bien de produire une éthique du dialogue, c'est-à-dire une éthique qui se fonde sur notre rapport à autrui. " La dimension éthique, écrit Fornet-Betancourt, de l'interculturalité en Amérique latine [cette dimension éthique ne se limite pas seulement à l'Amérique latine cependant] engage le dialogue interculturel vers les principes de libération et de justice. » (p. 168) II écrit dans un autre passage : "À ce stade, le dialogue interculturel se présente comme un projet éthique d'accueil de l'autre comme un être avec qui partager la souveraineté et un avenir qui ne soit pas déterminé uniquement par notre manière à nous de penser la vie. " (p. 150) Ce dernier passage n'est pas sans nous rappeler l'influence capitale d'Emmanuel Levinas sur la mise en place de la philosophie interculturelle. Accueillir autrui par le dialogue est ce vers quoi tend la philosophie interculturelle. Ce que nous avons décrit plus haut comme étant le travail continuel de l'éthique de l'interculturalité se confirme dans le passage suivant:

Nous devons souligner le fait que, malgré la coexistence de facto des cultures et les nombreux contacts existant entre elles, le dialogue interculturel - au sens fort que nous donnons à cette expression - est plus un « projet » qu'un fait avéré. En effet, nous devons reconnaître que, dans la phase actuelle de la globalisation néolibérale, le pouvoir de configurer la planète et de mener à terme cette configuration s'exerce à partir d'une stratégie d'homogénéisation d'un modèle de civilisation tellement imbu de sa supériorité qu'il relègue le dialogue interculturel à un niveau insignifiant ou contrôlé par ses propres intérêts. (p. 148-149)

Par ce constat de la situation actuelle, nous apercevons la tâche à accomplir pour mener à bien la visée d'un dialogue interculturel : il faut produire une philosophie interculturelle. C'est à cette tâche que nous convie FornetBetancourt. 
L'auteur en arrive rapidement à la conclusion suivante : pour réussir à décrire une philosophie interculturelle, c'està-dire décrire une philosophie dont le principal objectif est le dialogue entre les cultures, nous devons décentraliser la tradition européocentriste de la philosophie. " « Déphilosopher la philosophie » signifie la libérer de la prison où l'enferme la tradition hégémonique actuelle, à savoir la tradition occidentale européocentriste. 》(p. 169) Ou encore: "Il s'ensuit que déphilosopher la philosophie signifie en finir avec le préjugé qui voudrait que la philosophie soit un produit de la culture occidentale et dénoncer le caractère monoculturel de la définition ou de l'acceptation dominante de la philosophie. ॥ (p. 170) Cette critique du caractère monoculturel de la philosophie s'explique aisément du fait que la tradition philosophique est principalement tournée vers elle-même et qu'elle produit une réflexion qui est valide seulement dans le contexte européen. Comme n'importe quelle discipline académique, la philosophie existe à partir de ses propres règles de fonctionnement et à partir d'un champ limité de possibilités, par exemple la lecture obligatoire de certains textes dits fondateurs (La République de Platon, Le discours de la méthode de Descartes, La critique de la raison pure de Kant, Être et temps d'Heidegger). "À ce niveau, écrit Fornet-Betancourt, déphilosopher la philosophie, c'est la libérer de l'obligation de n'observer exclusivement que les lois d'un seul système du savoir ou d'un système éducatif déterminé. Déphilosopher renvoie ici à l'idée de dé-discipliner la philosophie, c'est-à-dire la sortir des limites imposées par les programmes d'études qui, depuis l’Europe centrale, se sont répandus dans le monde, et du rôle que lui attribue le système éducatif hégémonique. Plaider pour une philosophie dégagée de l'académisme revient à défendre une philosophie qui se pratique en dehors des contraintes et des règles imposées par le geôlier. » (p. 170) Fornet-Betancourt ne critique pas cette centralisation du savoir philosophique pour en contester la valeur, mais bien plutôt pour en contester le pouvoir globalisateur. Cette centralisation de la philosophie a pour conséquence majeure de laisser dans l'ombre l'existence d'autres traditions philosophiques présentes en dehors de l'Europe et de l'Amérique du Nord. Pour Fornet-Betancourt, il n'y a tout simplement pas de place pour l'existence d'une autre tradition philosophique en philosophie.

Cette fermeture du champ de la pensée traditionnelle en philosophie est causée également, selon FornetBetancourt, par son caractère académique. Ce caractère académique se rattache fondamentalement à la centralisation du savoir philosophique. Pour Fornet-Betancourt, déphilosopher la philosophie équivaut aussi bien à une décentralisation qu'à une sortie du monde académique. Fornet-Betancourt écrit à ce sujet :

Et cette réduction de la philosophie en une discipline académique, logiquement soumise aux intérêts sociaux et culturels incarnés par la formation dispensée dans les établissements "d'enseignement national ou public ", a peut-être eu comme conséquence la plus grave le fait que la philosophie soit restée prisonnière d'une tradition toute-puissante. Cette dernière s'érige en centre exclusif de tout développement futur et, entre autres choses, substitue l'étude des textes à la confrontation pratico-réflexive, c'est-à-dire au va-et-vient entre réflexion et situations concrètes de la vie. Pour cette tradition en effet - la tradition occidentale européenne -, les textes sont réellement considérés comme des dogmes, des principes sacrés, garants d'orthodoxie, et servent de modèle de base. (p. 30)

Fornet-Betancourt érige ainsi deux oppositions générales : premièrement, l'opposition entre un savoir académique et un savoir qui se déploie dans la pratique quotidienne, et deuxièmement, l'opposition entre la centralisation du savoir philosophique et la décentralisation de ce savoir dans le but de libérer la parole de ceux qui vivent en-dehors de la tradition philosophique occidentale.

Ces deux oppositions se conjuguent dans une même visée : contextualiser le savoir philosophique. «La philosophie est plurielle, parce qu'elle se pratique en de multiples langues, mais aussi parce qu'elle est une activité contextuelle. » (p. 30) Fornet-Betancourt ajoute: « Le savoir contextuel est un savoir qui s'articule aux processus historiques donnés. » (p. 30) Pour Fornet-Betancourt, le savoir philosophique doit être un savoir pratique qui se déploie dans un contexte historique précis. La philosophie interculturelle se veut ainsi un savoir qui cherche à rendre compte de la réalité de chaque espace culturel. "Avoir conscience que la transformation de la réalité, écrit Fornet-Betancourt, est l'enjeu du conflit explique le fait que le savoir philosophique contextuel se veut fondamentalement pratique. C'est-à-dire, encore une fois, un savoir-faire qui vise la réalité. » (p. 31) La philosophie interculturelle cherche à décrire la réalité de chaque culture en développant un savoir pratique. En insistant sur le caractère pratique du savoir philosophique interculturel, Fornet-Betancourt vise une double critique: critique interne de la tradition philosophique européenne d'une part et critique externe " de la réalité économique, sociale, politique, culturelle et religieuse de notre temps » (p. 29) d'autre part. Nous sommes ainsi au cœur de la onzième 
thèse des "Thèses sur Feuerbach » de Marx : «Les philosophes n'ont fait qu'interpréter le monde de différentes manières, ce qui importe c'est de le transformer. » (Marx, 1845/1968, p. 38)

La recherche de la description de la réalité de chaque culture ne se déploie pas pour autant en tant que repliement sur soi de chaque culture. Pour Fornet-Betancourt, c'est cette quête de la réalité de chaque culture qui se joue dans la philosophie interculturelle. Le dialogue, que nous avons décrit plus haut, est donc au centre de la recherche de la réalité. Il faut s'ouvrir à l'autre et confronter ainsi sa propre culture à la culture d'autrui pour en arriver à une meilleure compréhension de notre propre culture. Fornet-Betancourt écrit :

L'anti-centrisme de la "philosophie interculturelle » ne renvoie nullement à une négation ou à une déconsidération de l'environnement culturel propre et respectif. Bien au contraire, nous voudrions insister sur l'esprit critique qu'il convient d'avoir à l'égard de notre propre culture, sur le fait de ne pas sacraliser celle-ci ni céder à ses tendances ethnocentriques. II faut partir de sa propre tradition culturelle, en la considérant toutefois non pas comme une institution absolue, mais comme un lieu de passage vers l'intercommunication. (p. 43)

La décentralisation ne vise pas ainsi uniquement le savoir philosophique européen, mais bien la réalité de chaque culture. Le dialogue entre les cultures est donc le lieu où se joue l'intercommunication dans laquelle chaque culture s'ouvre à l'autre. La philosophie interculturelle vise ainsi la pluralité des voix et non le monologue d'une seule culture. Fornet-Betancourt met de l'avant autant «la polyphonie du logos philosophique » (p. 45) que la pluralité des voix culturelles.

Cette visée interculturelle se veut ainsi une ouverture et un accueil des voix et des cultures qui ont été jusqu'ici laissées de côté par la philosophie de tradition européenne. Fornet-Betancourt fait particulièrement référence à la situation de l'Amérique du Sud. Il écrit :

Notre objectif est la transformation de la raison, c'est-à-dire le souci d'introduire dans sa structuration les voix de ceux qui, jusqu'à présent, ont été " affectés » par les différents processus et qui malgré tout ne peuvent y prendre, une part active. La transformation de la raison, ou de la rationalité, signifierait ici l'incorporation égalitaire du point de vue du Sud dans la perception du monde et de l'histoire. C'est-à-dire qu'il s'agirait d'un projet de développement d'une rationalité dans laquelle la manière de comprendre le Sud ne serait plus quelque chose d'étrange, mais juste une approche légitime du monde et de I'histoire. (p. 48)

La philosophie interculturelle vise ainsi à donner une voix à ceux qui n'ont jamais pris part, à partir de leur propre culture, à un dialogue avec les autres cultures, à ceux qui ont été tenus au silence par la culture européoaméricaine. Fornet-Betancourt écrit finalement à propos de la tâche de la philosophie interculturelle :

II s'agit de formuler une proposition théorique et pratique pour une nouvelle transformation de la philosophie qui ne soit pas envisagée comme une simple tâche interne à la philosophie, une fin en soi, mais plutôt comme une initiative visant à mettre la philosophie à la hauteur des exigences réelles du contexte actuel de notre monde globalisé, afin qu'elle soit à même de mieux remplir sa fonction critique et libératrice. (p. 177)

Nous voyons, à partir de l'ouvrage de Fornet-Betancourt, que ce dernier vise à redécouvrir l'Amérique, mais cette fois une Amérique qui est autre que celle découverte par les Européens; une Amérique qui possède une autre voix, une voix plurielle.

\section{Références bibliographiques}

Derrida, J. (1994). Politiques de l'amitié. Paris : Galilée.

Marx, K. (1845/1968). "Thèses sur Feuerbach ». Dans L'idéologie allemande (H. Auger, G. Badia, J. Baudrillard et R. Cartelle, trad.). Paris : Éditions Sociales. 